\title{
Two new simple iridoids from the ant-plant Myrmecodia tuberosa and their antimicrobial effects
}

Nguyen Phuong Hanh ${ }^{\mathrm{a}}$, Nguyen Huu Toan Phan ${ }^{\mathrm{b}}$, Nguyen Thi Dieu Thuan ${ }^{\mathrm{b}}$, Tran Thi Hong Hanh $^{\mathrm{c}}$, Le Thi Vien ${ }^{\mathrm{c}}$, Nguyen Phuong Thao ${ }^{\mathrm{c}}$, Nguyen Van Thanh ${ }^{\mathrm{c}}$, Nguyen Xuan Cuong ${ }^{\mathrm{c}}$, Nguyen Quoc Binh ${ }^{\mathrm{d}}$, Nguyen Hoai Nam ${ }^{\mathrm{c}}$, Phan Van Kiem ${ }^{\mathrm{c}}$, Young Ho Kim ${ }^{\mathrm{e}}$ and Chau Van $\operatorname{Minh}^{\mathrm{c}, *}$

${ }^{a}$ Institute of Ecology and Biological Resources, Vietnam Academy of Science and Technology (VAST), 18 Hoang Quoc Viet, Caugiay, Hanoi, Vietnam; ${ }^{b}$ Tay Nguyen Institute for Scientific Research, VAST, 116 Xo Viet Nghe Tinh street, Dalat, Vietnam; ${ }^{c}$ Institute of Marine Biochemistry, VAST, 18 Hoang Quoc Viet, Caugiay, Hanoi, Vietnam; ${ }^{d}$ Vietnam National Museum of Nature, VAST, 18 Hoang Quoc Viet, Caugiay, Hanoi, Vietnam; ${ }^{e}$ College of Pharmacy, Chungnam National University, Daejeon 305-764, Republic of Korea.

*Corresponding author. Email: cvminh@ vast.vn

Six iridoid derivatives (1-6), including two new compounds myrmecodoides A and B (1 and 2), were isolated from the ant-plant Myrmecodia tuberosa. Their structures were determined on the basis of spectroscopic data $\left({ }^{1} \mathrm{H}\right.$ and ${ }^{13} \mathrm{C}$ NMR, HSQC, HMBC, ${ }^{1} \mathrm{H}-{ }^{1} \mathrm{H}$ COSY, NOESY, and HR-ESI-MS) and by comparison with the literature values. Among isolates, $\mathbf{3}$ and $\mathbf{4}$ exhibit weak antibacterial effect against Staphylococcus aureus subsp. aureus with MIC value of $100.0 \mu \mathrm{g} / \mathrm{mL}$.

Keywords: Myrmecodia tuberosa; Rubiaceae; ant-plant; iridoid; antimicrobial activity 


\section{CONTENTS}

\section{Pages}

$\begin{array}{ll}\text { Antimicrobial assays } & \text { S3 }\end{array}$

Figure S1. ${ }^{1} \mathrm{H}$ NMR spectrum $\left(\mathrm{CD}_{3} \mathrm{OD}, 500 \mathrm{MHz}\right)$ of myrmecodoide A (1) S5

Figure S2. ${ }^{13} \mathrm{C}$ NMR spectrum $\left(\mathrm{CD}_{3} \mathrm{OD}, 125 \mathrm{MHz}\right)$ of myrmecodoide $\mathrm{A}(\mathbf{1}) \quad$ S5

Figure S3. HSQC spectrum $\left(\mathrm{CD}_{3} \mathrm{OD}, 500 \mathrm{MHz}\right)$ of myrmecodoide A (1) S6

Figure S4. COSY spectrum $\left(\mathrm{CD}_{3} \mathrm{OD}, 500 \mathrm{MHz}\right)$ of myrmecodoide A (1) S6

Figure S5. $\mathrm{HMBC}$ spectrum $\left(\mathrm{CD}_{3} \mathrm{OD}, 500 \mathrm{MHz}\right)$ of myrmecodoide A (1) S7

Figure S6. NOESY spectrum $\left(\mathrm{CD}_{3} \mathrm{OD}, 500 \mathrm{MHz}\right)$ of myrmecodoide A (1) S7

Figure S7. ${ }^{1} \mathrm{H}$ NMR spectrum $\left(\mathrm{CD}_{3} \mathrm{OD}, 500 \mathrm{MHz}\right)$ of myrmecodoide B (2) S8

Figure S8. ${ }^{13} \mathrm{C}$ NMR spectrum $\left(\mathrm{CD}_{3} \mathrm{OD}, 125 \mathrm{MHz}\right)$ of myrmecodoide $\mathrm{B}(\mathbf{2}) \quad \mathrm{S} 8$

Figure S9. HSQC spectrum $\left(\mathrm{CD}_{3} \mathrm{OD}, 500 \mathrm{MHz}\right)$ of myrmecodoide $\mathrm{B}$ (2) S9

Figure S10. COSY spectrum $\left(\mathrm{CD}_{3} \mathrm{OD}, 500 \mathrm{MHz}\right)$ of myrmecodoide $\mathrm{B}(\mathbf{2}) \quad \mathrm{S} 9$

Figure S11. HMBC spectrum $\left(\mathrm{CD}_{3} \mathrm{OD}, 500 \mathrm{MHz}\right)$ of myrmecodoide $\mathrm{B}(2) \quad \mathrm{S} 10$

Figure S12. NOESY spectrum $\left(\mathrm{CD}_{3} \mathrm{OD}, 500 \mathrm{MHz}\right)$ of myrmecodoide B (2) S10

Figure S13. Key NOESY correlations of compounds 1 and $2 \quad$ S11

Table S1. ${ }^{1} \mathrm{H}\left(\mathrm{CD}_{3} \mathrm{OD}, 500 \mathrm{MHz}\right)$ and ${ }^{13} \mathrm{C} \mathrm{NMR}\left(\mathrm{CD}_{3} \mathrm{OD}, 125 \mathrm{MHz}\right) \quad \mathrm{S} 12$

spectroscopic data of compounds $\mathbf{1}$ and $\mathbf{2}$.

References

$\mathrm{S} 12$ 


\section{Antibacterial assay}

The antibacterial activity of the pure compounds was tested using the micro-dilution method on 96-well microtiter plate according to the method of Vanden Berghe and Vlietinck (1991) and McKane and Kandel (1996).

\section{Brieftly:}

Test microorganism strains:

+ Gram-(-) bacteria: Escherichia coli (ATCC 25922)

Pseudomonas aeruginosa (ATCC 25923 )

+ Gram-(+) bacteria: Bacillus subtilis (ATCC 11774 )

Staphylococcus aureus subsp. aureus (ATCC 11632)

+ Fungi: Aspergillus niger (439)

Fusarium oxysporum (M42)

+ Yeast: $\quad$ Candida albicans (ATCC 7754)

Saccharomyces cerevisiae (SH 20)

Positive controls:

$+\quad$ Streptomycin for $\mathrm{Gr}(+)$ bacteria

$+\quad$ Tetracycline for $\mathrm{Gr}(-)$ bacteria

$+\quad$ Nystatin or Amphotericin B for fungi and yeast.

Antibiotic agents was dissolved in DMSO 100\% with suitable concentrations.

Negative control:

Microorganisms without mixture of antibiotic agents and tested compounds.

Cultured media:

+ Media for maintain of strains: Sabouraud Dextrose Broth (SDB-Sigma) for fungi and yeast. Trypticase Soy Broth (TSB-Sigma) for bacteria.

$+\quad$ Tested media: Eugon Broth (Difco, USA) for bacteria, Mycophil (Difco, USA) for fungi and yeast.

Experiment:

+ The strains were activated and diluted according to McFarland 0.5 standard for experiment.

$+\quad$ The tested plates were incubated at $37^{\circ} \mathrm{C}$ for $24 \mathrm{hrs}$ with bacteria and at $30^{\circ} \mathrm{C}$ for 48 hrs with fungi and yeast. 
Calculation of the results:

Minimum inhibitory concentration (MIC) of tested samples: The samples were diluted with decrease of concentrations to reach minimum inhibitory concentration (MIC), the lowest concentration of each sample showing no growth.

The crude samples with MIC $\leq 200 \mu \mathrm{g} / \mathrm{ml}$ and pure compounds with $\mathrm{MIC} \leq 100$ $\mu \mathrm{g} / \mathrm{ml}$ were considered to be active. 


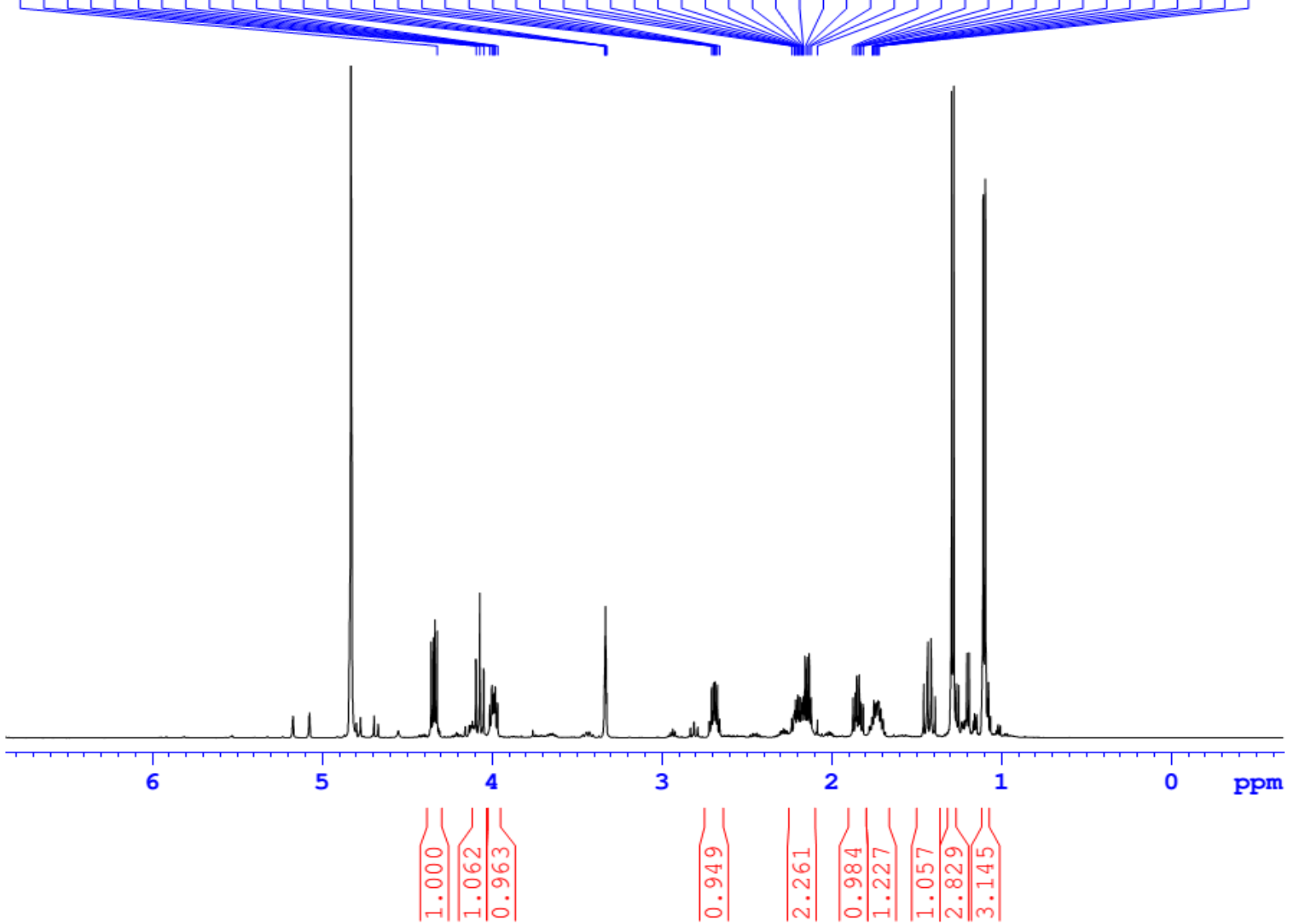

Figure S1. ${ }^{1} \mathrm{H}$ NMR spectrum $\left(\mathrm{CD}_{3} \mathrm{OD}, 500 \mathrm{MHz}\right)$ of myrmecodoide A (1)
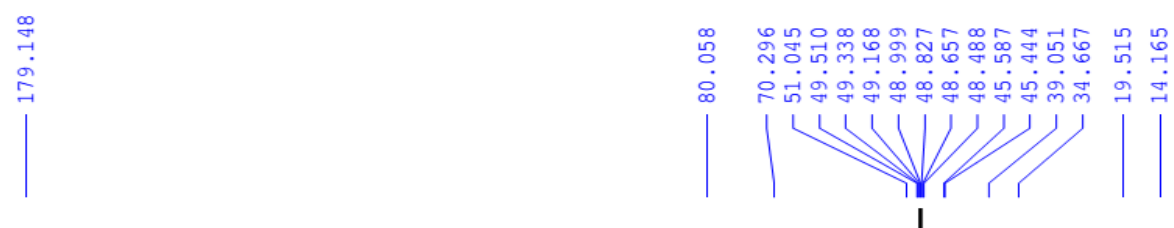
เ
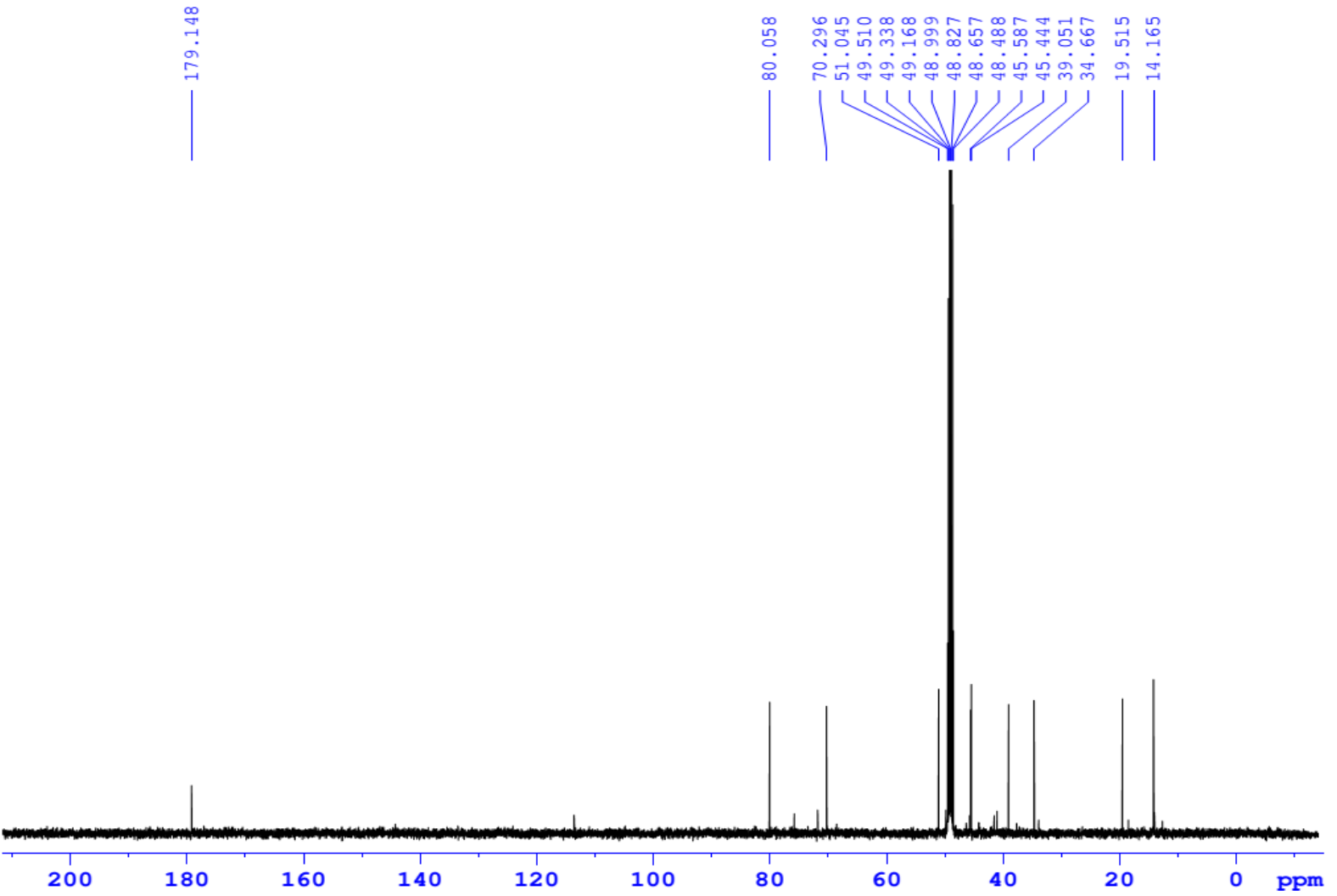

Figure S2. ${ }^{13} \mathrm{C}$ NMR spectrum $\left(\mathrm{CD}_{3} \mathrm{OD}, 125 \mathrm{MHz}\right)$ of myrmecodoide $\mathrm{A}(\mathbf{1})$ 


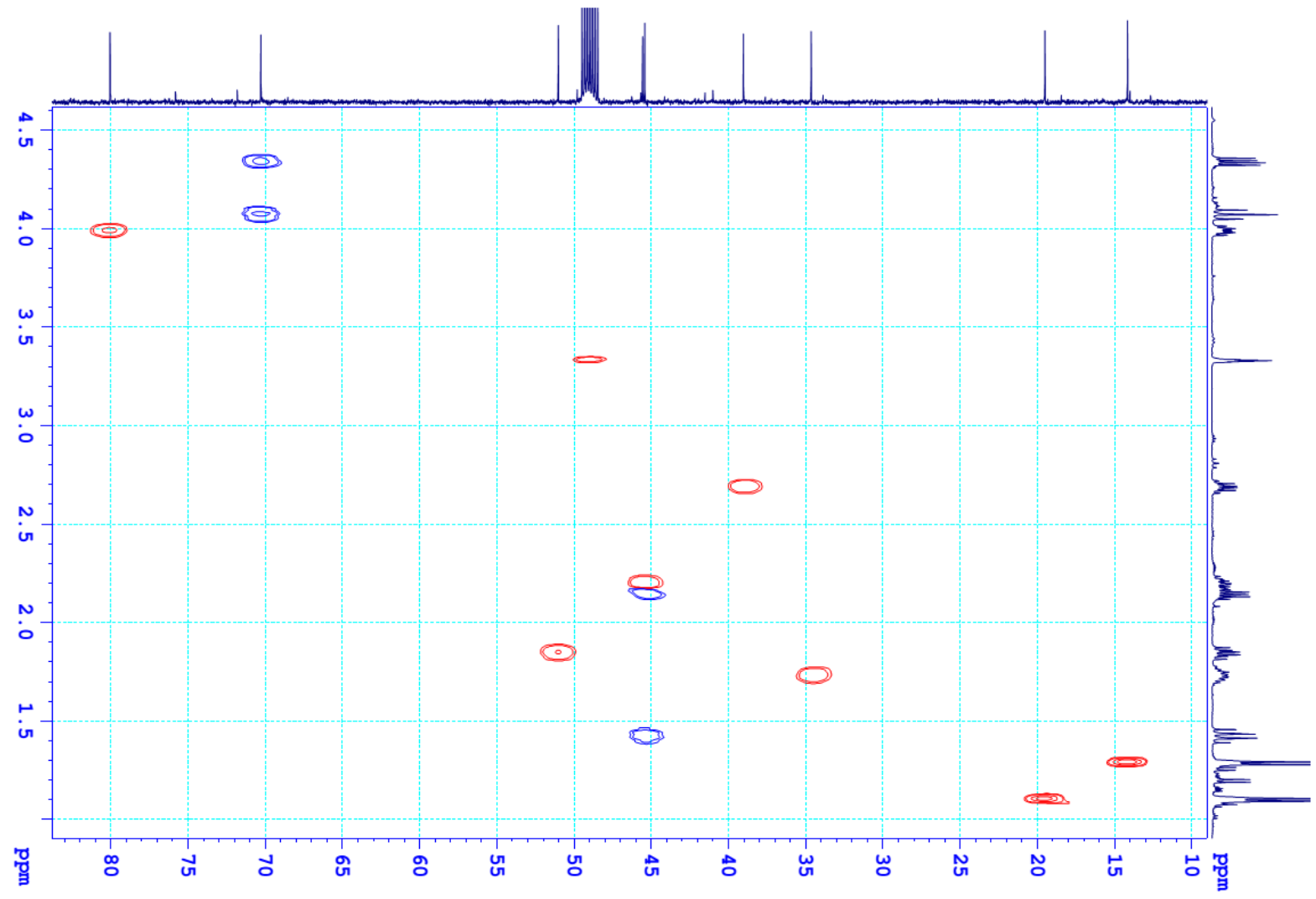

Figure S3. HSQC spectrum $\left(\mathrm{CD}_{3} \mathrm{OD}, 500 \mathrm{MHz}\right)$ of myrmecodoide A (1)

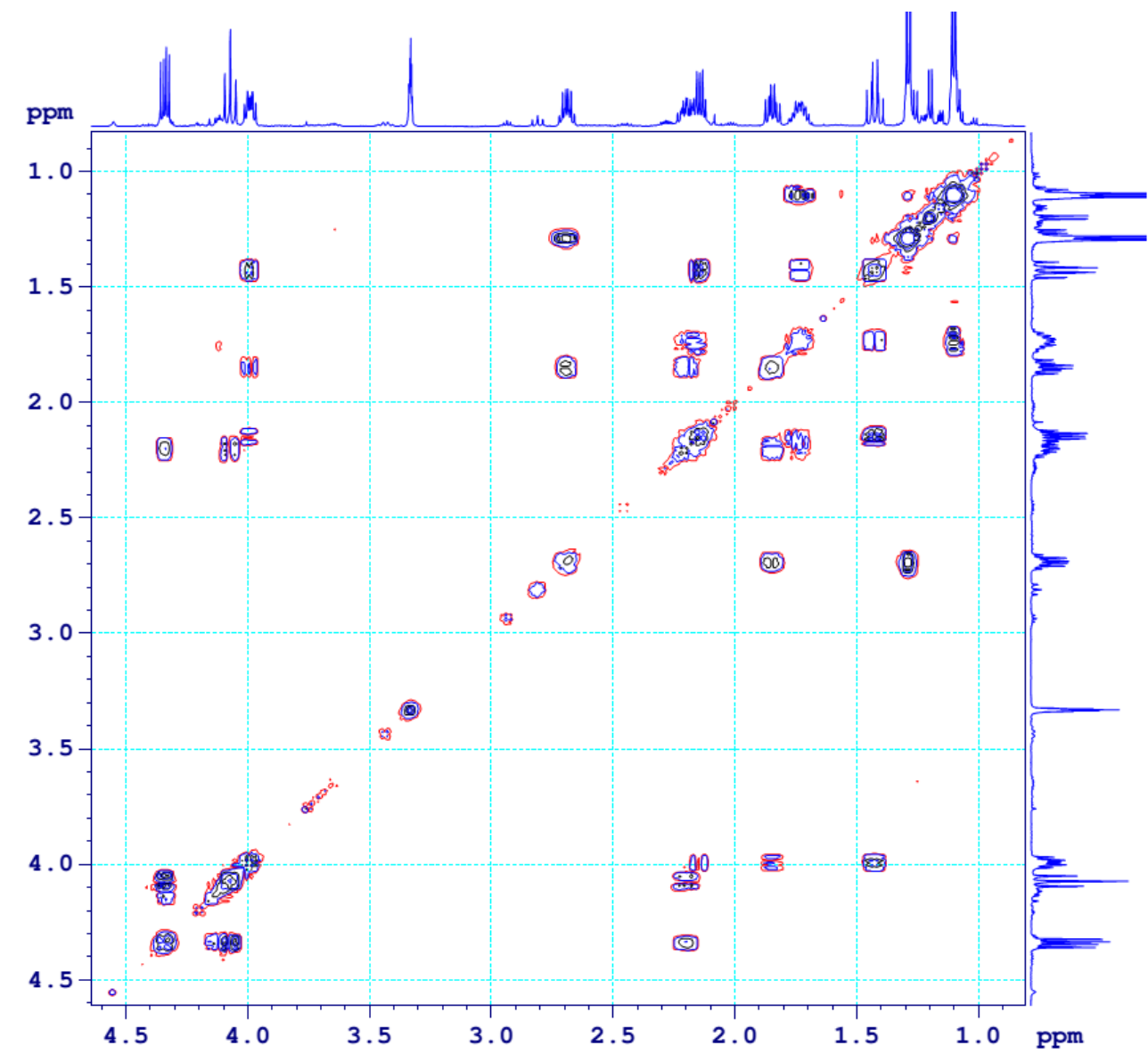

Figure S4. COSY spectrum $\left(\mathrm{CD}_{3} \mathrm{OD}, 500 \mathrm{MHz}\right)$ of myrmecodoide A (1) 


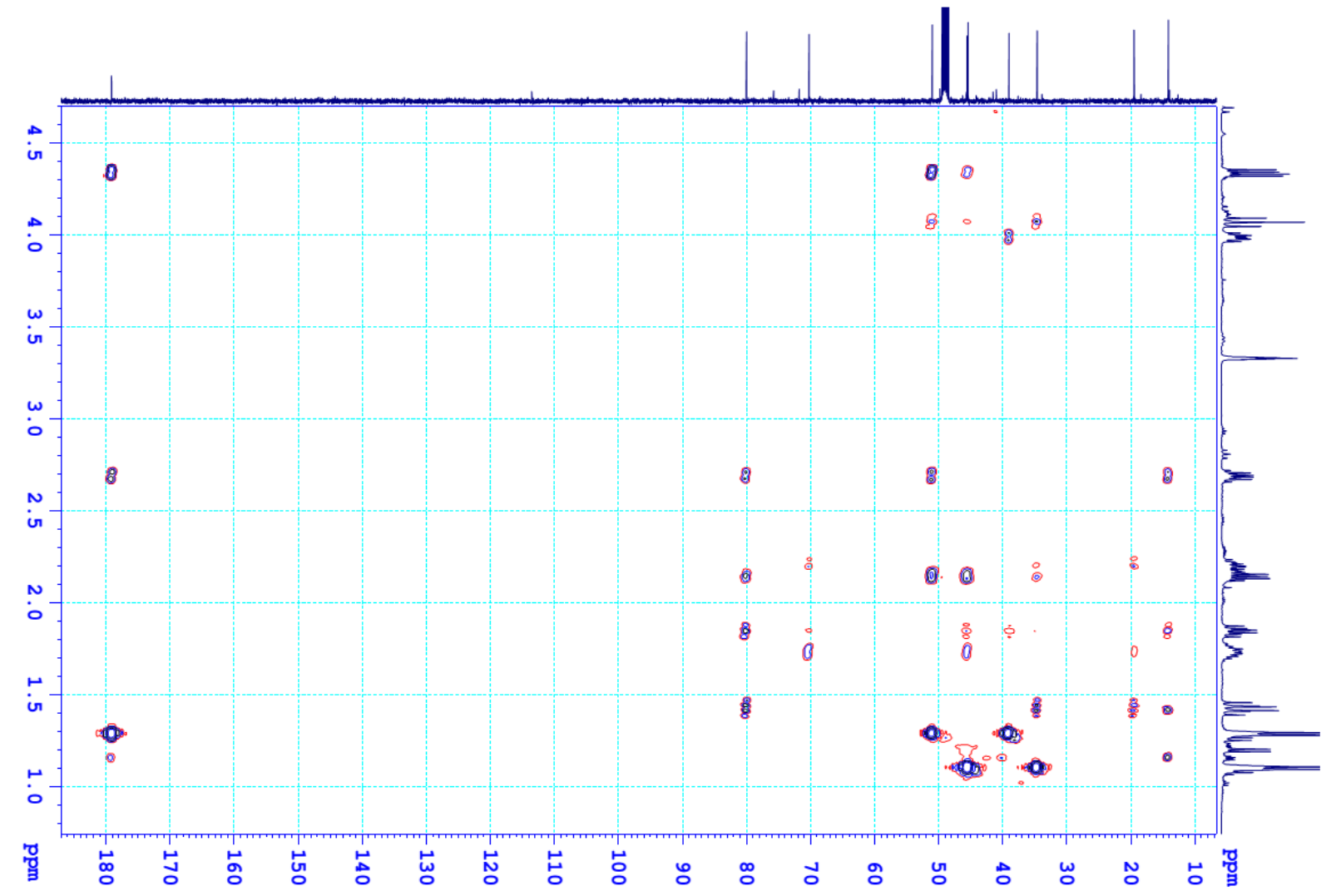

Figure S5. HMBC spectrum $\left(\mathrm{CD}_{3} \mathrm{OD}, 500 \mathrm{MHz}\right)$ of myrmecodoide A (1)

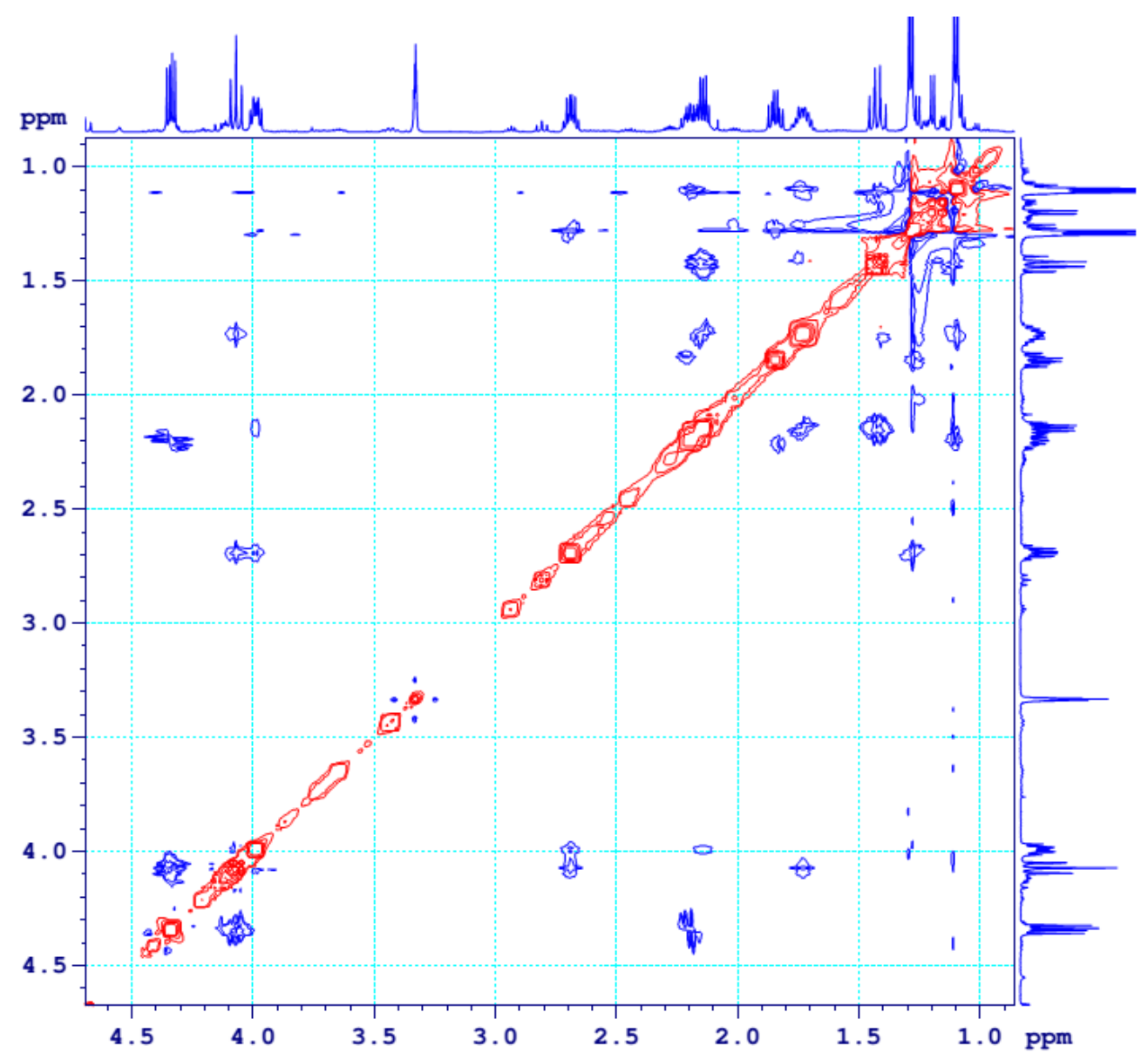

Figure S6. NOESY spectrum $\left(\mathrm{CD}_{3} \mathrm{OD}, 500 \mathrm{MHz}\right)$ of myrmecodoide A (1) 


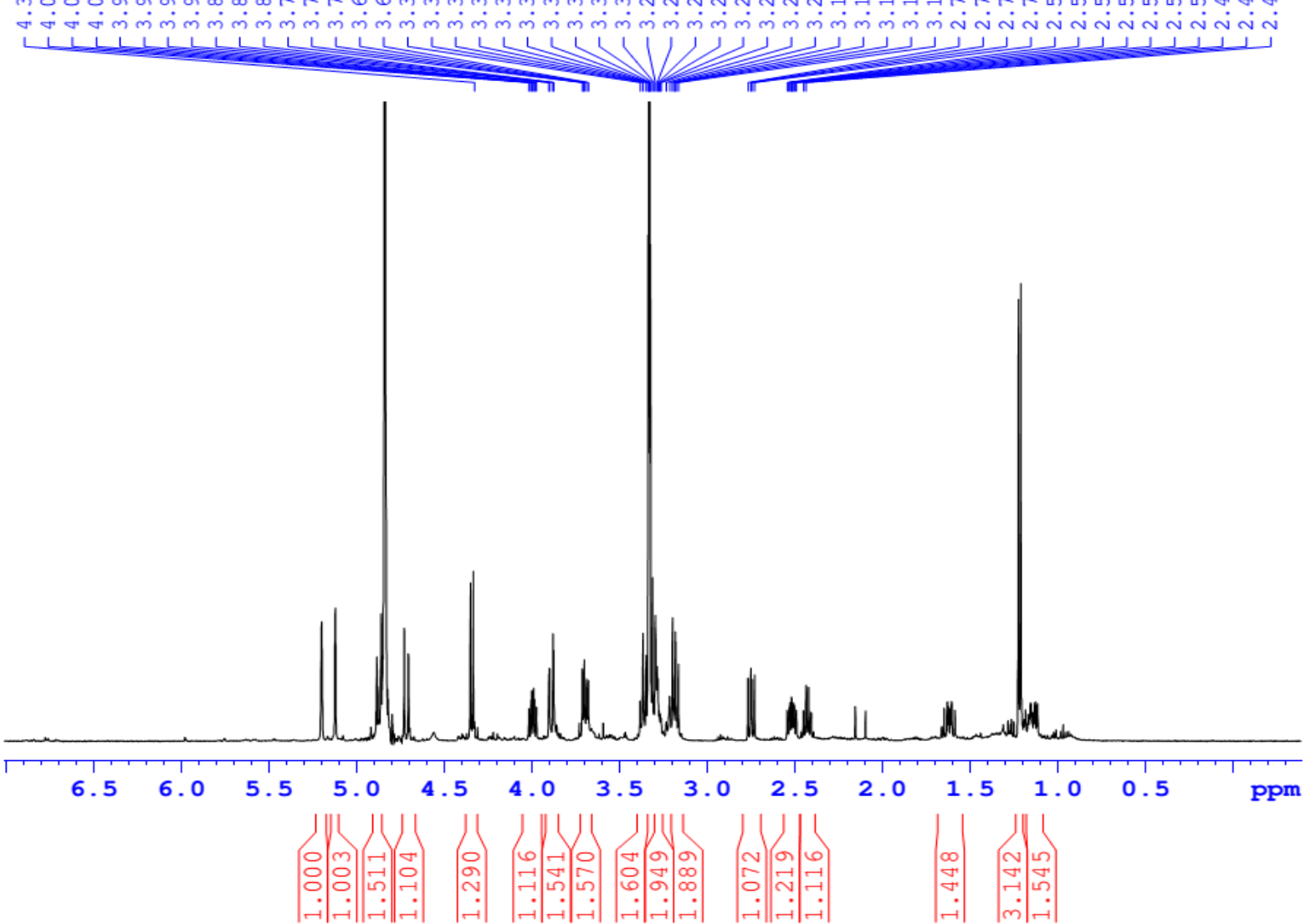

Figure S7. ${ }^{1} \mathrm{H}$ NMR spectrum $\left(\mathrm{CD}_{3} \mathrm{OD}, 500 \mathrm{MHz}\right)$ of myrmecodoide B (2)
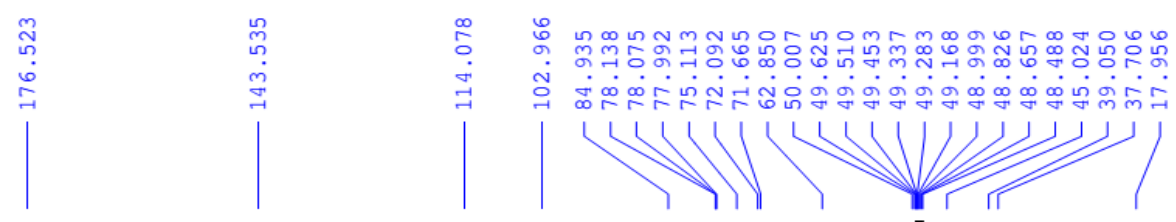

gure S8. ${ }^{13} \mathrm{C}$ NMR spectrum $\left(\mathrm{CD}_{3} \mathrm{OD}, 125 \mathrm{MHz}\right)$ of myrmecodoide $\mathrm{B}(\mathbf{2})$ 


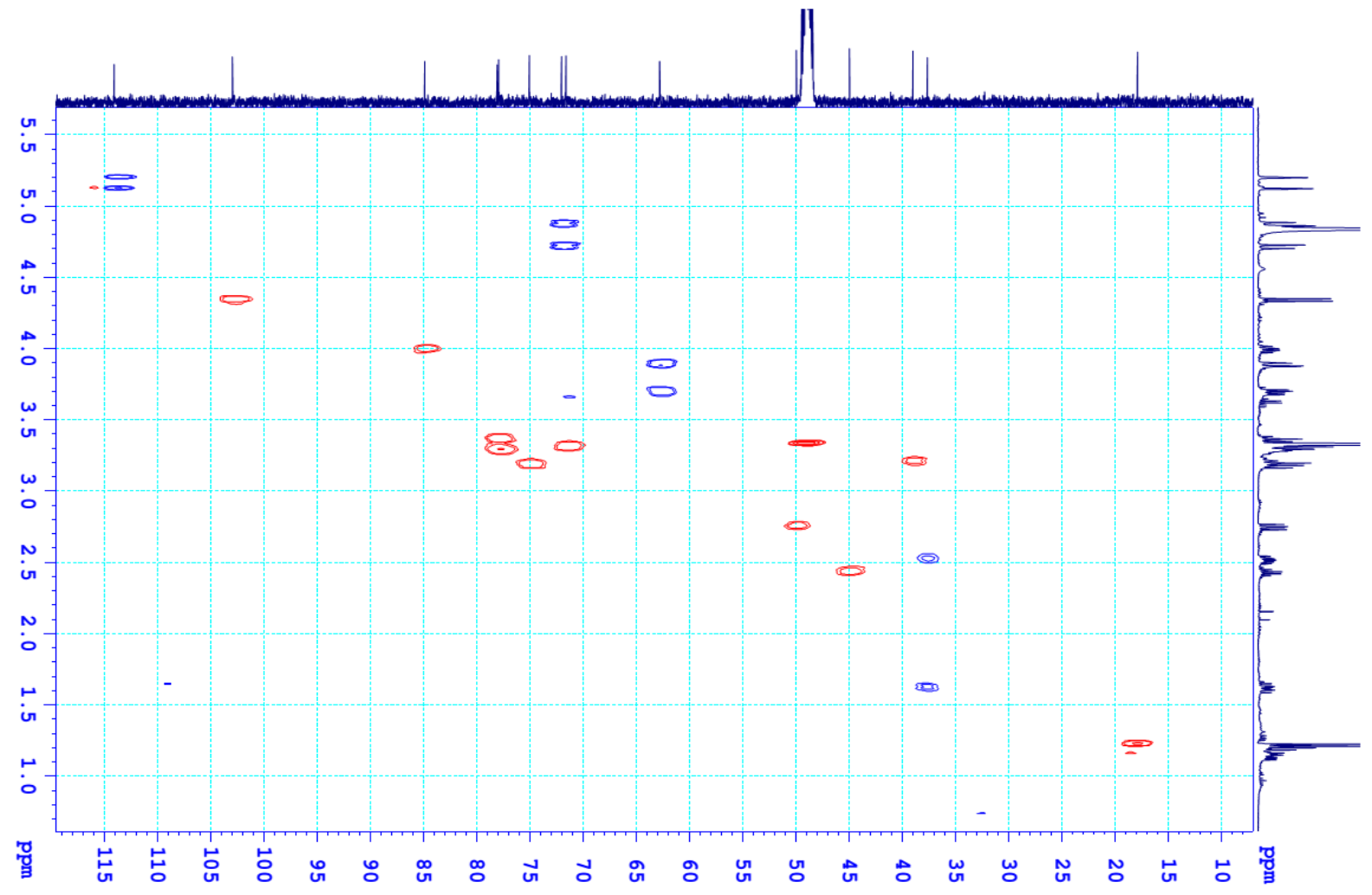

Figure S9. HSQC spectrum $\left(\mathrm{CD}_{3} \mathrm{OD}, 500 \mathrm{MHz}\right)$ of myrmecodoide $\mathrm{B}(2)$

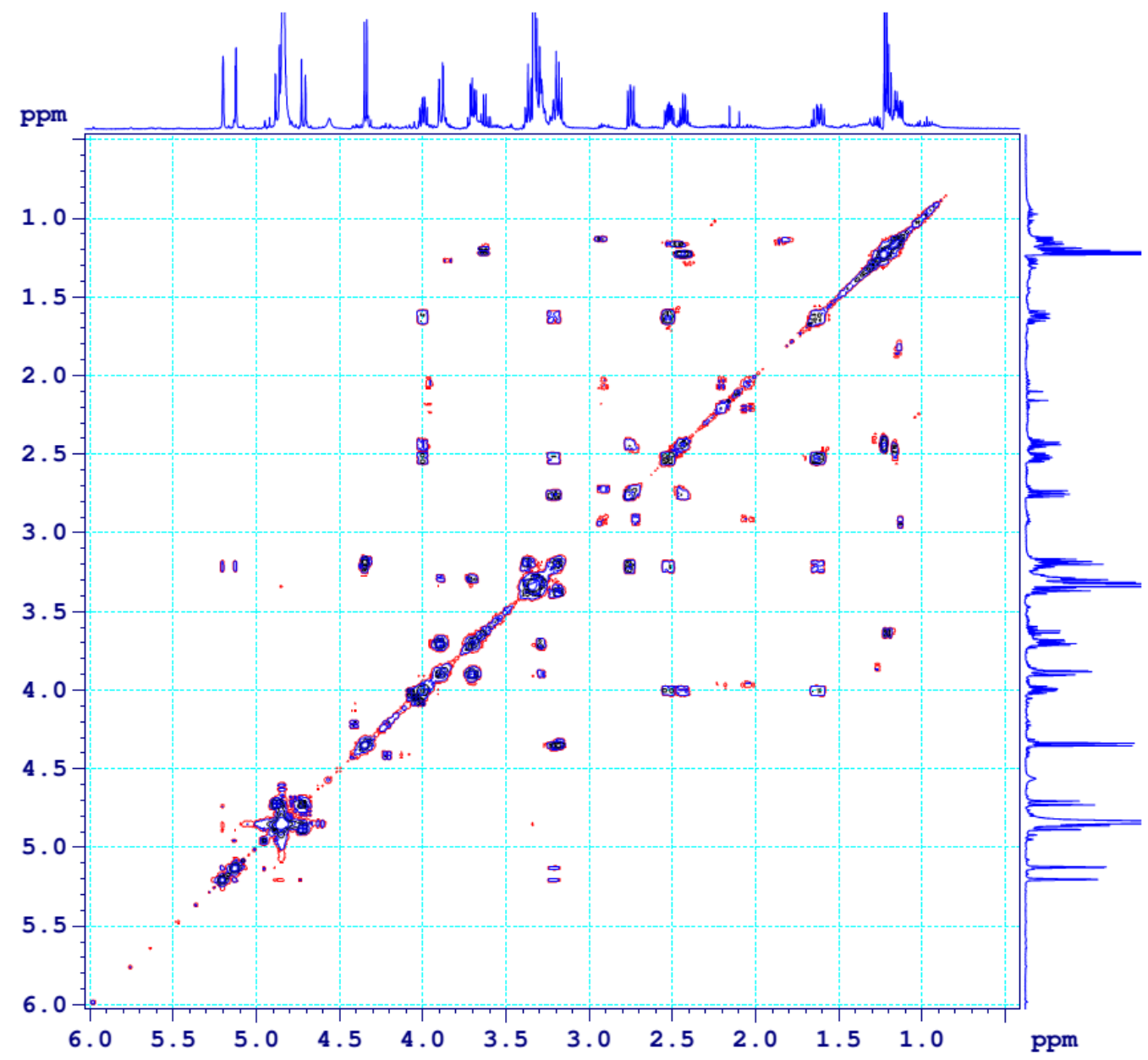

Figure S10. COSY spectrum $\left(\mathrm{CD}_{3} \mathrm{OD}, 500 \mathrm{MHz}\right)$ of myrmecodoide B (2) 


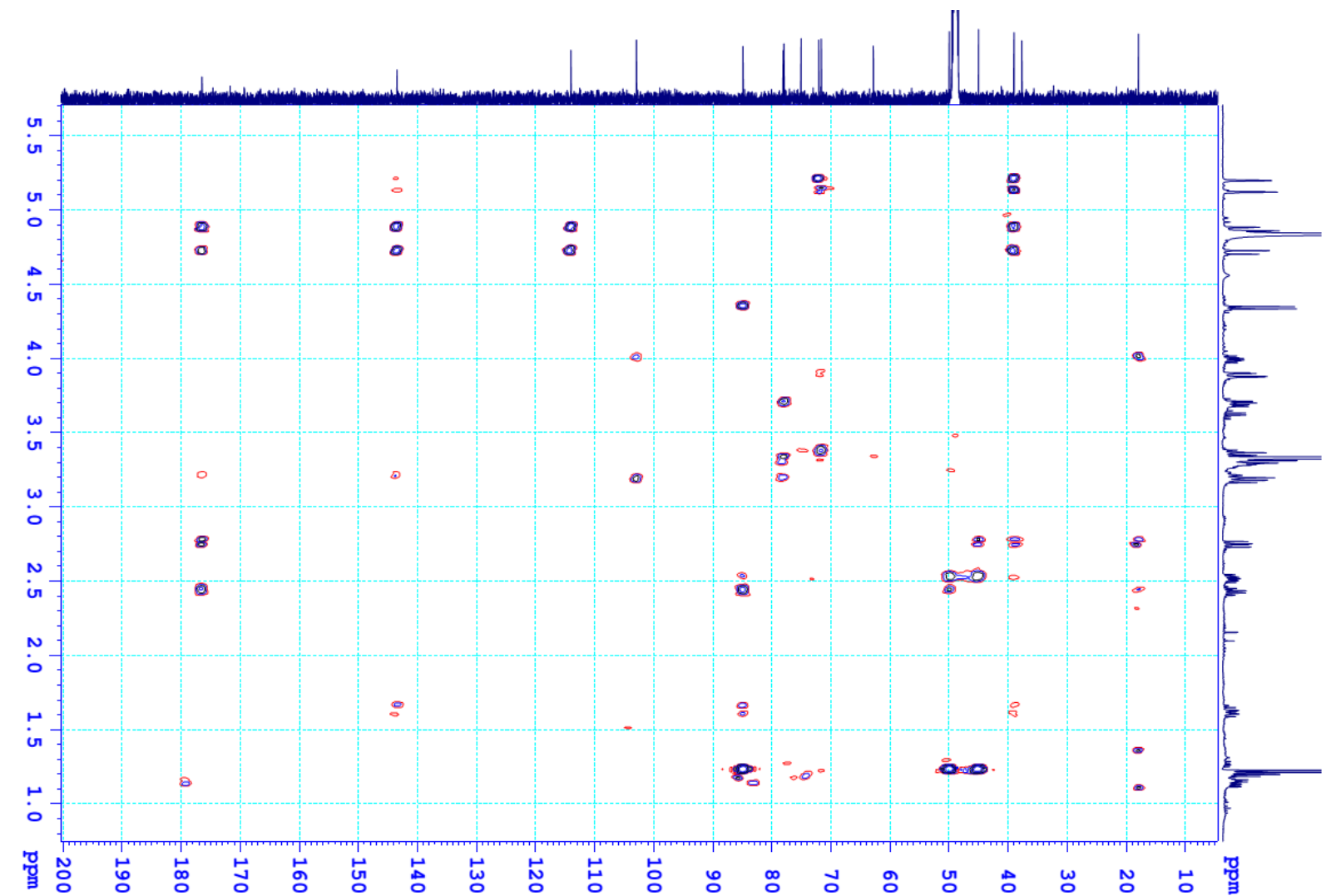

Figure S11. HMBC spectrum $\left(\mathrm{CD}_{3} \mathrm{OD}, 500 \mathrm{MHz}\right)$ of myrmecodoide B (2)

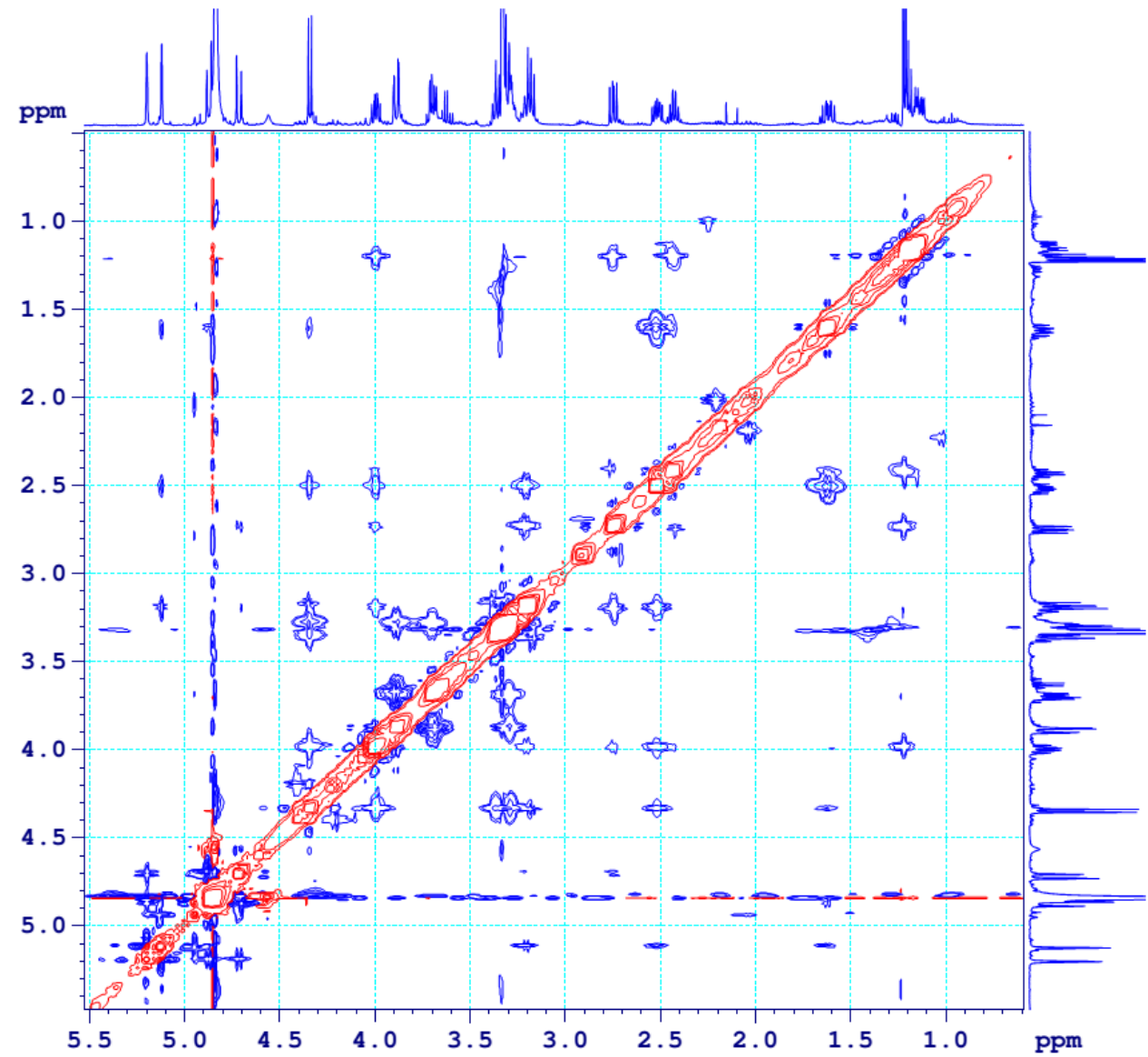

Figure S12. NOESY spectrum $\left(\mathrm{CD}_{3} \mathrm{OD}, 500 \mathrm{MHz}\right)$ of myrmecodoide $\mathrm{B}$ (2) 

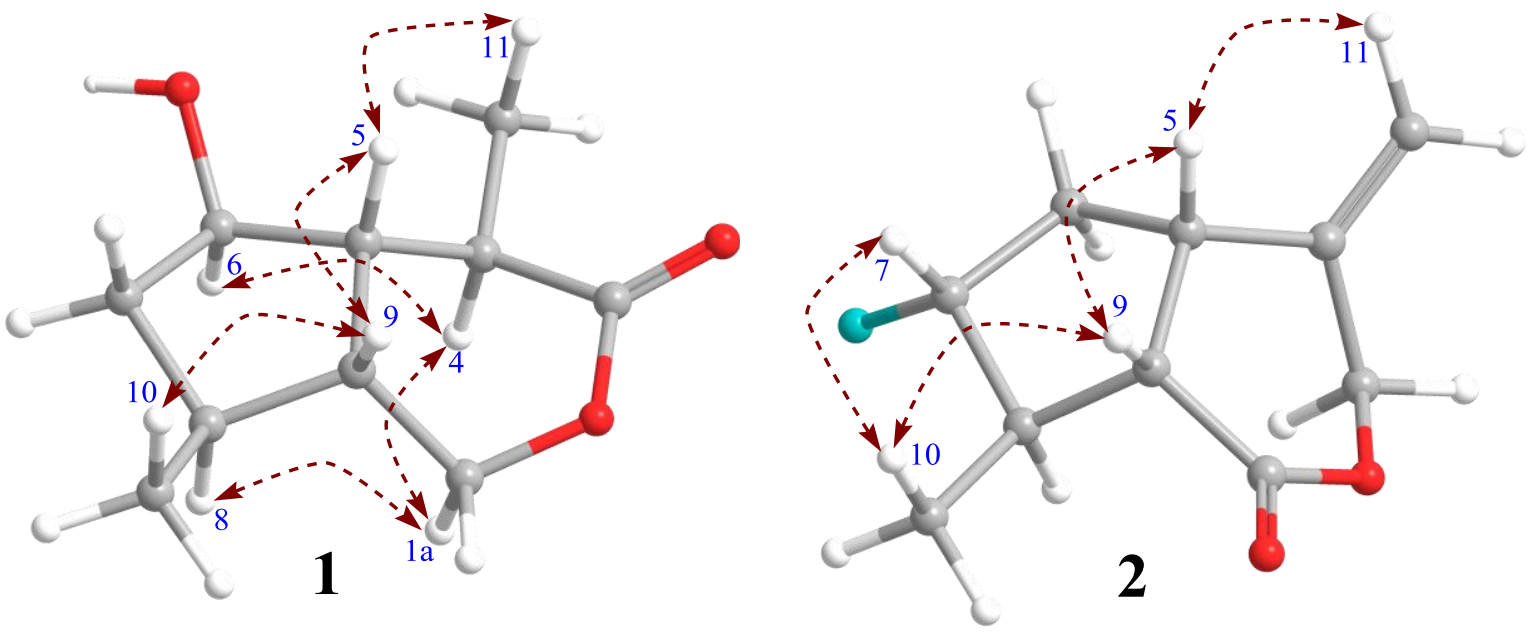

Figure S13. Key NOESY correlations of compounds 1 and 2 
Table S1. ${ }^{1} \mathrm{H}\left(\mathrm{CD}_{3} \mathrm{OD}, 500 \mathrm{MHz}\right)$ and ${ }^{13} \mathrm{C}$ NMR $\left(\mathrm{CD}_{3} \mathrm{OD}, 125 \mathrm{MHz}\right)$ spectroscopic data of compounds 1 and 2.

\begin{tabular}{|c|c|c|c|c|c|c|c|c|}
\hline \multirow[b]{2}{*}{ Pos. } & \multicolumn{4}{|l|}{1} & \multicolumn{4}{|l|}{2} \\
\hline & $\delta_{C}^{a, b}$ & $\delta_{\mathrm{H}}^{\mathrm{a}, \mathrm{c}}$ mult. $(J$ in $\mathrm{Hz})$ & ${ }^{1} \mathrm{H}-{ }^{1} \mathrm{H} \mathrm{COSY}$ & $\mathrm{HMBC}(\mathrm{H} \rightarrow \mathrm{C})$ & $\delta_{\mathrm{C}}^{\mathrm{a}, \mathrm{b}}$ & $\delta_{\mathrm{H}}^{\mathrm{a}, \mathrm{c}}$ mult. $(J$ in $\mathrm{Hz})$ & ${ }^{1} \mathrm{H}-{ }^{1} \mathrm{H}$ COSY & $\mathrm{HMBC}(\mathrm{H} \rightarrow \mathrm{C})$ \\
\hline 1 & 70.3 & $\begin{array}{l}4.07 \mathrm{dd}(11.0,11.5) \\
4.34 \mathrm{dd}(6.5,11.5)\end{array}$ & 9 & $3,5,8$ & 176.5 & - & & \\
\hline 3 & 179.1 & - & & & 72.1 & $\begin{array}{l}4.71 \mathrm{dd}(1.0,12.0) \\
4.87 \mathrm{brd}(12.0)\end{array}$ & & $1,4,5,11$ \\
\hline 4 & 39.0 & $2.68 \mathrm{dq}(11.0,7.0)$ & 5,11 & $3,5,6,11$ & 143.5 & - & & \\
\hline 5 & 51.0 & $1.84 \mathrm{ddd}(7.0,10.5,11.0)$ & $4,6,9$ & $1,4,11,6,9$ & 39.0 & $3.21 \mathrm{~m}$ & 6,9 & 1,4 \\
\hline 6 & 80.0 & $3.99 \mathrm{ddd}(6.0,10.5,11.0)$ & 5,7 & 4 & 37.7 & $1.62 \mathrm{~m} / 2.51 \mathrm{~m}$ & 5,7 & $4,5,7,8,9$ \\
\hline 7 & 45.4 & $\begin{array}{l}1.42 \mathrm{ddd}(9.5,11.0,12.0) \\
2.14 \mathrm{dd}(6.0,12.0)\end{array}$ & 6,8 & $6,8,10$ & 84.9 & 4.00 ddd $(6.0,8.0,10.0)$ & 6,8 & $10,1^{\prime}$ \\
\hline 8 & 34.7 & $1.73 \mathrm{~m}$ & $7,9,10$ & 1,10 & 45.0 & $2.43 \mathrm{ddq}(8.0,10.5,7.0)$ & $7,9,10$ & $1,7,9,10$ \\
\hline 9 & 45.6 & $2.21 \mathrm{~m}$ & $1,5,8$ & 1,10 & 50.0 & $2.74 \mathrm{dd}(8.0,10.5)$ & 5,8 & $1,5,8,10$ \\
\hline 10 & 19.5 & $1.09 \mathrm{~d}(7.0)$ & 8 & $7,8,9$ & 17.9 & $1.21 \mathrm{~d}(7.0)$ & 8 & $7,8,9$ \\
\hline 11 & 14.2 & $1.28 \mathrm{~d}(7.0)$ & 4 & $3,4,5$ & 114.1 & $\begin{array}{l}5.12 \mathrm{~d}(1.5) \\
5.20 \mathrm{br} \mathrm{s}\end{array}$ & & $3,4,5$ \\
\hline $1^{\prime}$ & & & & & 102.9 & $4.34 \mathrm{~d}(8.0)$ & $2^{\prime}$ & 7 \\
\hline $2^{\prime}$ & & & & & 75.1 & $3.19 \mathrm{dd}(8.0,9.0)$ & $1^{\prime}, 3^{\prime}$ & \\
\hline $3^{\prime}$ & & & & & 78.1 & $3.67 \mathrm{t}(9.0)$ & $2^{\prime}, 4^{\prime}$ & \\
\hline $4^{\prime}$ & & & & & 71.7 & $3.30 \mathrm{t}(9.0)$ & $3^{\prime}, 5^{\prime}$ & \\
\hline $5^{\prime}$ & & & & & 78.0 & $3.28 \mathrm{~m}$ & $3^{\prime}, 6^{\prime}$ & \\
\hline $6^{\prime}$ & & & & & 62.8 & $\begin{array}{l}3.70 \mathrm{dd}(5.0,12.0) \\
3.88 \mathrm{dd}(2.0,12.0)\end{array}$ & $5^{\prime}$ & \\
\hline
\end{tabular}

Note: All assignments were done by HSQC, COSY, HMBC, and NOESY experiments

\section{References}

McKane L, Kandel J. 1996. Microbiology. New York: McGraw-Hill.

Vanden Berghe DA, Vlietinck AJ. 1991. Screening methods for antibacterial and antiviral agents from higher plants. In: Methods in Plant Biochemistry. London: Academic Press. p. 47-69. 